\title{
Personalization of Multi-electrode Setups in tCS/tES: Methods and Advantages
}

\author{
R. Salvador, M. C. Biagi, O. Puonti, M. Splittgerber, V. Moliadze, \\ M. Siniatchkin, A. Thielscher, and G. Ruffini
}

\section{Introduction}

Transcranial current stimulation (tCS), including transcranial direct current stimulation (tDCS), transcranial alternating current stimulation (tACS), and transcranial random noise stimulation (tRNS), is a family of noninvasive neuromodulatory techniques that employ weak (1-4 mA) electrical currents applied via electrodes placed on the scalp for long durations (20-40 min) [1, 2]. Concurrent effects of stimulation range from changes in cortical excitability [3] to modulation of ongoing endogenous oscillations [4]. Hebbian-based mechanisms are hypothesized to lead to long-lasting plastic changes in the brain [5], leading to an increasing interest in putative therapeutic applications in a range of neurological diseases [6]. One factor that limits the usefulness of tCS is the widely reported intersubject variability of

R. Salvador $(\bowtie) \cdot$ M. C. Biagi

Neuroelectrics, Barcelona, Spain

e-mail: ricardo.salvador@neuroelectrics.com

O. Puonti - A. Thielscher

Danish Research Centre for Magnetic Resonance, Centre for Functional and Diagnostic Imaging and Research, Copenhagen University Hospital Hvidovre, Hvidovre, Denmark

Department of Health Technology, Technical University of Denmark, Kongens Lyngby, Denmark

M. Splittgerber · V. Moliadze

Institute of Medical Psychology and Medical Sociology, University Medical Center Schleswig Holstein, Kiel University, Kiel, Germany

M. Siniatchkin

Clinic of Child and Adolescent Psychiatry and Psychotherapy, Evangelic Hospital Bethel

(EvKB), Bielefeld, Germany

G. Ruffini

Neuroelectrics, Barcelona, Spain

Starlab, Barcelona, Spain

(C) The Author(s) 2021 
responses to stimulation [7]. Several factors can explain variability, but here we will focus on the physical agent of the effects that tCS has on neurons: the electric field (E-field) induced in the tissues.

\subsection{Biophysical Aspects of tCS}

The distribution of currents in the head can be described mathematically by the electric field (E-field) vector induced by tCS. Depending on the orientation of the latter with respect to neuronal processes, the membrane of pyramidal neurons is polarized (approximately $0.2 \mathrm{mV}$ per $1 \mathrm{~V} / \mathrm{m}$ of E-field value, [8]), which leads to the observed concurrent effects of stimulation. One common hypothesized mechanism is the polarization of the soma of pyramidal cells due to the component of the E-field perpendicular to the cortical surface $\left(E_{n}\right)$, [9]. However, other mechanisms of interaction are possible, such as the polarization of axon terminals [10].

The E-field distribution depends on factors such as head geometry (thickness and shape of the head tissues), electrical properties of the tissues (electrical conductivity, $\sigma)$, location and geometry of the electrodes, and the currents that are applied via the electrodes [11]. Since in vivo measurements of the E-field still pose a number of technical challenges $[12,13]$ and cannot easily be carried out, computational head models based on structural data (usually head MRIs) are usually employed to estimate it $[14,15]$.

Initial uses of computational head models were limited to a posteriori analysis of the E-field distribution of electrode montages typically applied in experimental protocols [15, 16]. In recent years, several algorithms have been described to leverage these head models with the objective of optimizing some dose parameters (position and currents of the stimulating electrodes) to target a specific brain region and/or cortical network [9, 17-20].

This paradigm shift from "one-model-fits-all" montages to individualized montages leveraging subject-specific head models and dose optimization algorithms has the potential to reduce intersubject variability in the outcomes of tCS and allow for more effective and safe protocols. However, several parameters can affect the outcome of these modeling and optimization pipelines. In this work, we will study how uncertainties in target specification, tissue electrical conductivities, and the threshold for neuromodulatory effects can affect the outcome of the optimization. We will also discuss some of the potential benefits of these pipelines, especially in relation to reduction of intersubject variability of the results of optimization. 


\section{Methods}

\subsection{Subjects}

We included seven healthy children and adolescents (three males) aged 10-17 years (M 14; SD 2). The study was approved by the Ethics Committee of the Faculty of Medicine, Kiel University, Kiel Germany. ${ }^{1}$ All participants and their parents were instructed about the study, and written informed consent according to the Declaration of Helsinki on biomedical research involving human subjects was obtained. The study is part of the STIPED project. ${ }^{2}$

\subsection{Head Model Generation}

Each subject underwent structural head scanning on a 3 T Philips Achieva scanner, during which the following sequences were acquired: a T1-weighted scan $\left(1 \mathrm{~mm}^{3}\right.$, $\mathrm{TR}=2530 \mathrm{~ms}, \mathrm{TE}=3.5 \mathrm{~ms}, \mathrm{TI}=1100 \mathrm{~ms}, \mathrm{FA}=7^{\circ}$, fast water excitation), a T2-weighted scan $\left(1 \mathrm{~mm}^{3}, \mathrm{TR}=3200 \mathrm{~ms}, \mathrm{TE}=300 \mathrm{~ms}\right.$, no fat suppression), and a diffusion MRI (dMRI) scan $\left(2 \mathrm{~mm}^{3}, \mathrm{TR}=6300 \mathrm{~ms}, \mathrm{TE}=51 \mathrm{~ms}, 67\right.$ directions, $\mathrm{b}=1000)$.

Tissue segmentation was performed using an in-house implementation combining extra-cerebral tissue segmentations from a new segmentation approach, which will be included in a future version of the open-source simulation toolbox SimNIBS, ${ }^{3}$ with brain tissue segmentations and cortical gray matter (GM) surface reconstructions from FreeSurfer [21]. Finite element head models were then generated (see Fig. 1), including representations of the scalp, skull, cerebrospinal fluid (CSF), gray matter, and white matter (WM). The head models also contained representations of Pistim electrodes $\left(1 \mathrm{~cm}\right.$ radius, cylindrical $\mathrm{Ag} / \mathrm{AgCl}$ electrodes $\left.{ }^{4}\right)$ placed in 61 positions of the 10-10 EEG system. For the electrodes, only the conductive gel underneath the metal connector was represented in the head model. Unless otherwise stated, the scalp, skull, and CSF were modeled as isotropic with conductivities of $0.33 \mathrm{~S} / \mathrm{m}, 0.008 \mathrm{~S} / \mathrm{m}$, and $1.79 \mathrm{~S} / \mathrm{m}$, respectively, which are appropriate values for the DC-low frequency values used in tCS [15]. The GM and WM were modeled as anisotropic (volume normalization, [22], with isotropic conductivity values used for diffusion tensor scaling of $0.40 \mathrm{~S} / \mathrm{m}-0.15 \mathrm{~S} / \mathrm{m}$, for the $\mathrm{GM}-\mathrm{WM}$, [15]). E-field calculations were performed in $\mathrm{COMSOL}^{5}$ using secondorder tetrahedral mesh elements to solve Laplace's equation [11].

\footnotetext{
${ }^{1}$ http://apps.who.int/trialsearch/Trial2.aspx?TrialID=DRKS00008207

${ }^{2} \mathrm{http}: / /$ www.stiped.eu/home/

${ }^{3}$ https://simnibs.github.io/simnibs/build/html/index.html

${ }^{4}$ www.neuroelectrics.com

${ }^{5}$ v5.3, www.comsol.com
} 
Fig. 1 Finite element head model generated for one of the subjects in this study.

The model includes representations of the scalp (in yellow), skull (in gray), CSF (in blue), GM (in gray), and WM (in white), as well as gel underneath the electrodes (in green). Air cavities are represented as cavities in the mesh, thus effectively modeling the air as an insulator

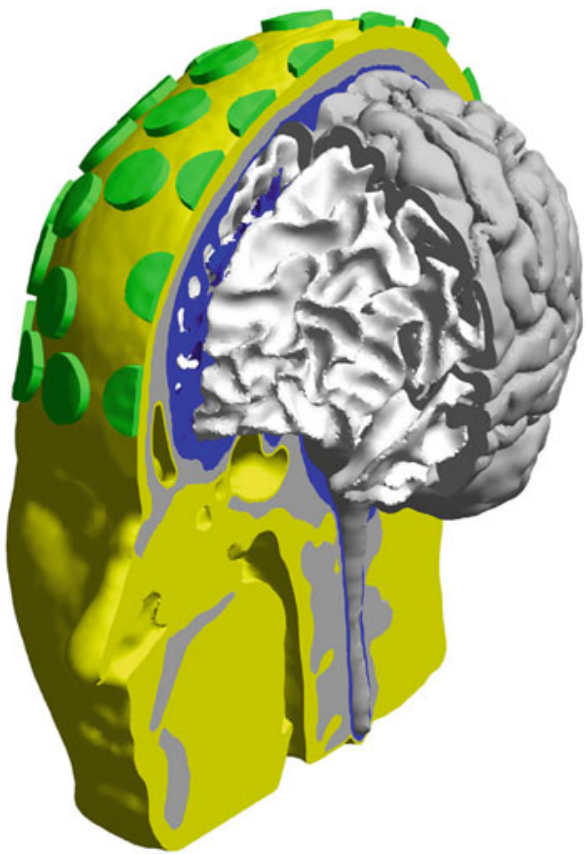

\subsection{Montage Optimization Algorithm}

The optimization algorithm used in this study is based on the Stimweaver algorithm [9]. We assumed the normal component of the E-field to the cortical (GM-CSF) surface $E_{n}$ as responsible for the acute effects of stimulation. Positive/negative $E_{n}$-values, corresponding to $E$-fields directed into/out-of-the cortical surface, lead to increased/decreased excitability of the soma of pyramidal cells. Inputs to this algorithm include target $E_{n}$-maps, with information about the target $E_{n}$-field $\left(E_{n}^{\text {Target }}\right)$ in each node of the cortical surface; weight maps, with information about the priority (weight, $w$ ) assigned to each node in the optimization; current constraints of the montage (maximum current, in absolute value, per channel $I_{\text {max }}$ channel $=\max _{i}\left\{\left|I_{i}\right|\right\}$ and maximum total injected current $I_{\text {max total }}=$ $\frac{1}{2} \sum_{i=1}^{N_{\text {Channels }}}\left|I_{i}\right|$ ); and the maximum number of electrodes in the montage. The objective function in the optimization is the error with respect to no intervention (ERNI, with units of $\mathrm{mV}^{2} / \mathrm{mm}^{2}$ ):

$$
E R N I=\sum_{i=1}^{N} \frac{\left(w_{i} \sum_{j=1}^{N_{\text {channels }}-1} E_{n, i}^{j-C z} I_{j}-w_{i} E_{n, i}^{\text {Target }}\right)^{2}-\left(w_{i} E_{n, i}^{\text {Target }}\right)^{2}}{\sum_{j=1}^{N} w_{j}^{2}}
$$

where $N$ is the number of mesh nodes, $N_{\text {channels }}$ is the number of electrodes available for the optimization, $E_{n, i}^{j-C z}$ is a column vector (lead-field vector) with the normal 
component of the E-field induced by a bipolar montage that has $j$ as the anode $(+1 \mathrm{~mA})$ and $C z$ as the cathode $(-1 \mathrm{~mA})$, and $I_{j}$ is the current (in $\mathrm{mA}$ ) of electrode $j$ in the montage that is being evaluated. The term $\sum_{j=1}^{N_{\text {Channels }}-1} E_{n, i}{ }^{j-C z} I_{j}$ yields the normal component of the E-field in the montage being evaluated (for each node $i$ ), as follows from the linearity principle [9]. The lead-field terms $E_{n, i}^{j-C z}$ are calculated on a subject-specific basis using the methods detailed in the previous section. The optimization without the constraint on the number of active electrodes was performed by in-house scripts programmed in Python using the SciPy library. ${ }^{6}$ In order to constrain the number of electrodes of the final montage, a genetic algorithm (GA) was implemented following the methods described in [9].

\subsection{Studies Performed}

In this work, we performed several studies to clarify the impact of several inputs and parameters to the optimization algorithm in the results. The first study (study $a$ ) aims at determining the impact of target size on the optimization results. This is related to the perceived mechanisms of stimulation underlying the effects of tCS, with some studies focusing on highly localized targets, obtained, for instance, from EEG source localization information [17, 20], where other studies focus on more widespread cortical areas with information extracted from cytoarchitectural information [23] or functional imaging data [24]. Optimization algorithms can tackle both of these cases, but it is unclear how current constraints influence the capability of achieving the desired target $E_{n}$-field with increasing target area size.

Another important parameter that affects the E-field distribution, and therefore the results of the optimization, is the electrical conductivity of the head tissues. Measuring these values in vivo still presents several limitations, and data available in the literature has a wide variability, representing different measuring methods and origin of the tissue samples [25]. Furthermore, some reports indicate that this value might change according to the subject's age, at least for the skull [26]. In study $b$, we partially tackled this problem by assessing the influence that different conductivity values for the skull tissue have on the optimization results.

In study $c$, we investigated some of the potential benefits of optimization algorithms on experimental design, namely, less variability on $E_{n}$-field distribution.

More details about how each study was performed are presented in the next section. In the studies where surface average $E_{n}$ values are presented, they were calculated with the following expression:

\footnotetext{
${ }^{6}$ https://www.scipy.org/scipylib/
} 


$$
<E_{n}>=\frac{\int_{\text {Area Patch }} E_{n} d A}{A_{\text {Patch }}}=\frac{\sum_{i=1}^{N_{\text {Patch }}} E_{n, i} A_{i}}{\sum_{i=1}^{N_{\text {Patch }}} A_{i}}
$$

where $A_{i}$ is the area associated with each node of the mesh (the sum of the areas of all the triangles connected to the node divided by 3 ) and $N_{\text {patch }}$ is the number of nodes in the surface patch where the average is being calculated.

\section{Results}

\subsection{Study A: Effect of Target Size}

In this study, we investigated how target size and current constraints affect the actual $<E_{n}>$ achievable on a target given the current constraints. The target was located on the left precentral gyrus, and its size varied from $9 \mathrm{~mm}^{2}$ (a tiny spot on the gyrus crown) to $\sim 275 \mathrm{~mm}^{2}$ (about the entire left frontal lobe). The different areas were first identified on the cortical surface of a template brain model (Colin $27^{7}$ ), starting from the smallest one and progressively enlarging it up to the maximum size considered. Each area was then remapped separately onto the cortical surface of one of the participants of this study, and single target maps were created: the target area was assigned to excitation, with two values of $E_{n}^{\text {Target }}(0.25 \mathrm{~V} / \mathrm{m}$ and $0.50 \mathrm{~V} / \mathrm{m})$, and maximum weight $w_{\text {stim }}=10$; the rest of the cortex was assigned no stimulation $\left(E_{n}^{\text {Target }}=0 \mathrm{~V} / \mathrm{m}\right)$ with weight $w_{\text {no-stim }}$ varying for each area, in such a way that both conditions have the same relative importance to the ERNI calculation: (wo-stim $)$ $\left.w_{\text {stim }}\right)^{2}=$ Area $_{\text {stim }} /$ Area $_{\text {no-stim }}$.

Figure 2 shows $\left\langle E_{n}>\right.$ on the target as a function of target size. The $E_{n}$ distribution results from optimized montages with an unconstrained number of electrodes, obtained for different combinations of $E_{n}{ }^{\text {Target }}$, maximum current per electrode, and total injected current $\left(I_{\max }\right.$ channel, $\left.I_{\max \text { total }}\right)$ including values exceeding the usual safety limit of $4.0 \mathrm{~mA}$.

For all current constraints and both $E_{n}{ }^{\text {Target }}$ values, we observe an overall logarithmic decrease of the $\left\langle E_{n}\right\rangle$ with the target size. This decrease is more rapid for the higher $E_{n}{ }^{\text {Target }}$, and it follows a non-monotonic trend that can be correlated to the cortical curvature of the target area and to the distance between the target area and electrodes. In fact, the significant drop at $108 \mathrm{~mm}^{2}$ w.r.t. the previous size is likely due to the fact that the ROI is now large enough to comprehend both faces of a sulcus, which have surface normals - and consequently normal electric field pointing in opposite directions: once averaged over the whole ROI, this results in

\footnotetext{
${ }^{7}$ https://www.bic.mni.mcgill.ca/ServicesAtlases/Colin27
} 


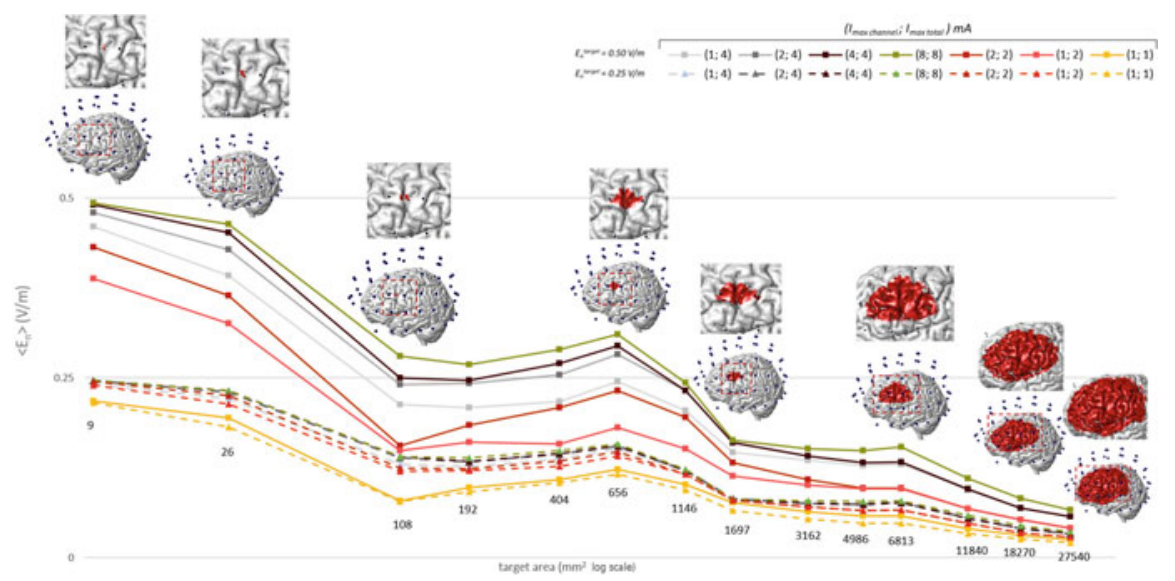

Fig. 2 Average normal component of the E-field on target (red area on the GM surfaces), as a function of the target area (in $\log$ scale), for different values of the target normal E-field $\left(E_{n}{ }^{\text {Target }}=0.25 \mathrm{~V} / \mathrm{m}\right.$ dashed lines, $E_{n}{ }^{\text {Target }}=0.50 \mathrm{~V} / \mathrm{m}$ continuous lines $)$ and combinations of the individual and total current constraints (yellow, $I_{\max }$ total $=2 \mathrm{~mA}$; pink and red, $I_{\text {max }}$ total $=2 \mathrm{~mA}$; gray and black, $I_{\max \text { total }}=4 \mathrm{~mA}$; green, $I_{\max \text { total }}=8 \mathrm{~mA}$ ). The pictures also show the available positions of the electrodes on the scalp

a decrease of $\left\langle E_{n}\right\rangle$. With the next area increase, the ROI extends out of the sulcus, over the two adjacent gyri, and approaches the normal projection of the center of the two closest electrodes, which is reflected in the slow increase of $\left\langle E_{n}\right\rangle$, reaching a local peak at $656 \mathrm{~mm}^{2}$. Further area increases, in the second half of the plot, repeat this pattern, ultimately created by the compounding effect of the gyrification of the target area and the distance from the covering electrodes.

Concerning the current constraints, in Fig. 3, we look separately at the influence of the $I_{\text {max total }}(3 \mathrm{a})$ and of the $I_{\text {max channel }}(3 \mathrm{~b})$. The shaded area in blue in Fig. 3a represents the value of the maximum $\left\langle E_{n}\right\rangle$ achievable in each target, for both $E_{n}{ }^{\text {Target }}$, normalized with respect to $E_{n}{ }^{\text {Target }}$. This maximum $\left\langle E_{n}\right\rangle$ is obtained with a montage optimization with unrestricted maximum individual and total injected current and is the same for both values of $E_{n}{ }^{\text {Target }}$. As we observe, it also decays logarithmically with the target area, from $95 \%{E_{n}}^{\text {Target }}$ for the smallest target to $15 \%$ $E_{n}^{\text {Target }}$ for the largest. In this case the decay is to attribute utterly to the effect of head anatomy and electrode positions. The figure also shows the $\left\langle E_{n}>\right.$ obtained with different current constraints, normalized with respect to the maximum $<E_{n}>$ achievable, for both $E_{n}^{\text {Target }}$.

We observe that, for $E_{n}^{\text {Target }}=0.50 \mathrm{~V} / \mathrm{m}$, only with a $I_{\max \text { total }}=8.0 \mathrm{~mA}$ it is possible to induce in all areas a $\left\langle E_{n}>\right.$ at least over $80 \%$ of the maximum $\left\langle E_{n}>\right.$ achievable. On the other hand, a total injected current $I_{\text {max total }}$ of $1.0 \mathrm{~mA}$ does not reach even the half of the maximum achievable $\left\langle E_{n}\right\rangle$, in any target, including the smallest. Moreover, we observe that, as a result of the linearity of $I_{\text {max total }}$ and $E_{n}{ }^{\text {Target }}$, for the less stringent condition of $E_{n}{ }^{\text {Target }}=0.25 \mathrm{~V} / \mathrm{m}$, the exact same relative $\left\langle E_{n}\right\rangle$ on each target area can be achieved with half of the total current. 


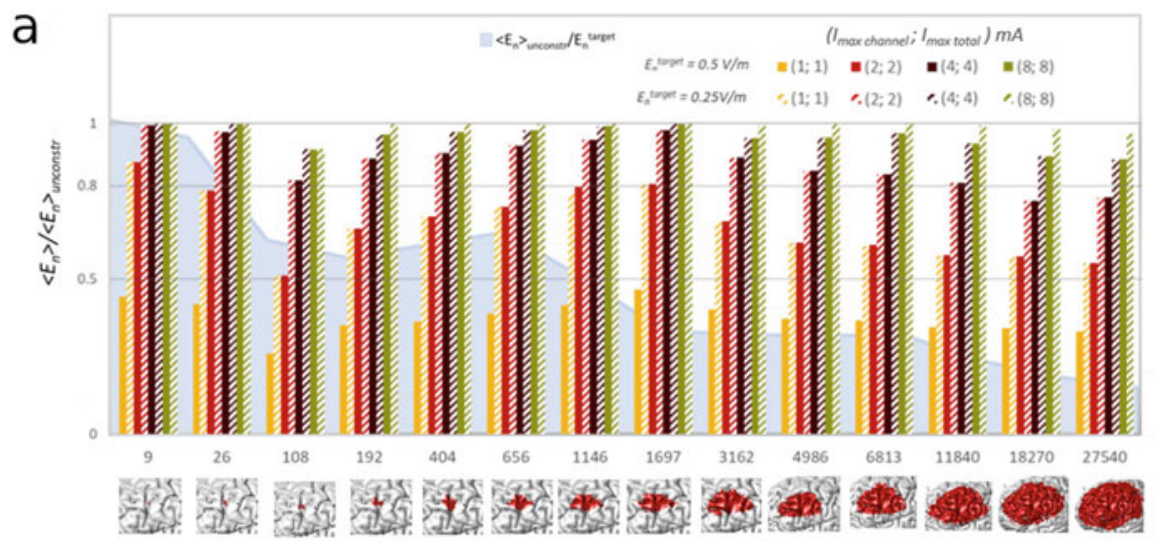

b

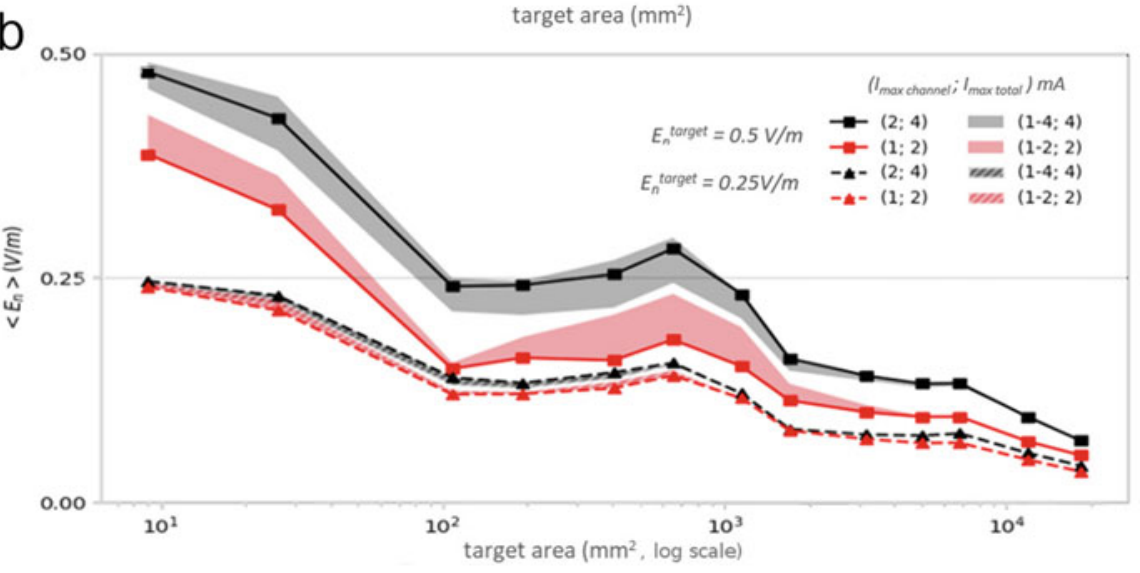

Fig. 3 Effect of the total injected (a) and individual (b) current constraints on $\left\langle E_{n}>\right.$ on target areas of different sizes, for different target $E_{n}$-fields. (a) The bars show the relative $\left\langle E_{n}>\right.$ w.r.t. the $\left\langle E_{n}\right\rangle$ calculated with unconstrained current, per each target area and $E_{n}{ }^{\text {Target }}$; the area shows the relative unconstrained $<E_{n}>$ w.r.t. $E_{n}^{\text {Target }}$ (same for both values of $E_{n}^{\text {Target }}$ ). (b) The shaded areas represent the $\left\langle E_{n}\right\rangle$ obtained with $I_{\text {max channel }}$ within $1 \mathrm{~mA}$ and $I_{\text {max total }}$, for $E_{n}{ }^{\text {Target }}=0.50 \mathrm{~V} / \mathrm{m}$ and $I_{\max }$ ${ }_{\text {total }}=4 \mathrm{~mA}$ (solid gray), $E_{n}^{\text {Target }}=0.50 \mathrm{~V} / \mathrm{m}$ and $I_{\text {max }}$ total $=2 \mathrm{~mA}$ (solid red), $E_{n}^{\text {Target }}=0.25 \mathrm{~V} / \mathrm{m}$ and $I_{\text {max total }}=4 \mathrm{~mA}$ (dashed gray), $E_{n}^{\text {Target }}=0.25 \mathrm{~V} / \mathrm{m}$ and $I_{\text {max total }}=4 \mathrm{~mA}$ (dashed red). The lines represent solutions with $I_{\text {max channel }}=I_{\text {max total }} / 2$, for $E_{n}^{\text {Target }}=0.50 \mathrm{~V} / \mathrm{m}$ (solid lines) and $E_{n}^{\text {Target }}=0.25 \mathrm{~V} / \mathrm{m}$ (dashed lines)

Consequently, $I_{\text {max total }}=4.0 \mathrm{~mA}$ in this case is sufficient to reach at least $80 \%$ of the maximum achievable $\left\langle E_{n}>\right.$. In Fig. $3 \mathrm{~b}$, we focus on the current constraints considered in studies b and c. This plot indicates that $I_{\text {max channel }}$ modulates the $\left\langle E_{n}\right\rangle$ only up to a given target size (which is smaller for the less demanding condition: $E_{n}^{\text {Target }}=0.25 \mathrm{~V} / \mathrm{m}$ ). After this threshold area, the only factor influencing $\left\langle E_{n}\right\rangle$ becomes $I_{\text {max }}$ total. 


\subsection{Study B: Tissue Conductivity Values}

In this study, we assessed how skull conductivity $\left(\sigma_{\text {skull }}\right)$ values affected the optimization results. We tried three different conductivity values: $0.008 \mathrm{~S} / \mathrm{m}$ (our standard conductivity value which corresponds to a ratio of scalp-to-skull conductivity of 41), $0.011 \mathrm{~S} / \mathrm{m}$ (scalp-to-skull conductivity ratio of 30), and $0.041 \mathrm{~S} / \mathrm{m}$ (ratio of 8 ). These values cover a wide range of values reported in the literature [26]. For one of the subjects in this study, we calculated the lead-field matrix and performed optimizations with a common target: the left dorsolateral prefrontal cortex (IDLPFC) as identified by Brodmann area 46 [27]. The cortical surface nodes in this area were set to a target $E_{n}$-value of either $0.25 \mathrm{~V} / \mathrm{m}$ or $0.50 \mathrm{~V} / \mathrm{m}$ with weight 10 . The remaining nodes were set to a target $E_{n}$-value of $0 \mathrm{~V} / \mathrm{m}$ with a weight of 2 . Current constraints were set to $\left(I_{\text {max channel }}, I_{\text {max total }}\right)=(2.0,4.0) \mathrm{mA}$ and $\left(I_{\text {max channel }}, I_{\text {max }}\right.$ total $)=(1.0,2.0) \mathrm{mA}$.

Figure 4 a displays $\left\langle E_{n}\right\rangle$ on the IDLPFC as a function of $\sigma_{\text {skull }}$ for the different current and target $E_{n}$ constraints. Average $E_{n}$ values increase nonlinearly with $\sigma_{\text {skull }}$ for every set of constraints except for the less stringent one: target $E_{n}$ of $0.25 \mathrm{~V} / \mathrm{m}$ with $\left(I_{\text {max channel }}, I_{\text {max total }}\right)=(2.0,4.0) \mathrm{mA}$. Figure $4 \mathrm{~b}$ displays the variation of the total injected current $\left(I_{\text {total }}\right)$ in each montage with $\sigma_{\text {skull }}$. $I_{\text {total }}$ tends to decrease nonlinearly with increasing $\sigma_{\text {skull }}$, except for the most stringent constraint: target $E_{n}$ of $0.50 \mathrm{~V} / \mathrm{m}$ with $\left(I_{\text {max channel }}, I_{\text {max total }}\right)=(1.0,2.0) \mathrm{mA}$. In this case, $I_{\text {total }}$ stays almost constant at the highest value allowed $(2.0 \mathrm{~mA})$.

Figure 5 provides the distribution of $E_{n}$ in the cortical surface for all the optimizations performed in this study. For all optimizations and for the highest $\sigma_{\text {skull }}$, the position of the electrodes in the optimized montage is very similar across the different sets of constraints, with only the current values being different. For lower $\sigma_{\text {skull }}$ values, and especially in the more stringent optimization constraints, the montages also differ in the electrode positions, often employing bigger separations between the anodes and cathodes.

We also evaluated the change in average $E_{n}$ value that would occur when the optimized montages were evaluated in a model with a different $\sigma_{\text {skull }}$ than the one used to derive the montage $\left(\sigma_{\text {skull }}^{\text {Eval }} \neq \sigma_{\text {skull }}{ }^{\text {Optim }}\right)$. This led to changes in average $E_{n}$ values ranging from $-45 \%$ to $137 \%$ of the values obtained when $\sigma_{\text {skull }}^{\text {Eval }}=\sigma_{\text {skull }}^{\text {Optim }}$. The average $E_{n}$ values decreased when $\sigma_{\text {skull }}^{\text {Eval }}<\sigma_{\text {skull }}{ }^{\text {Optim }}$ and they increased otherwise.

\subsection{Study C: Intersubject Variability}

In study $c$, we investigated the advantages that montage optimization brings in terms of intersubject variability of the E-field distribution. To do this, we performed subject-specific optimizations for six of the subjects in this study. For each subject, the optimization parameters were the same as the ones employed in study b. Each 

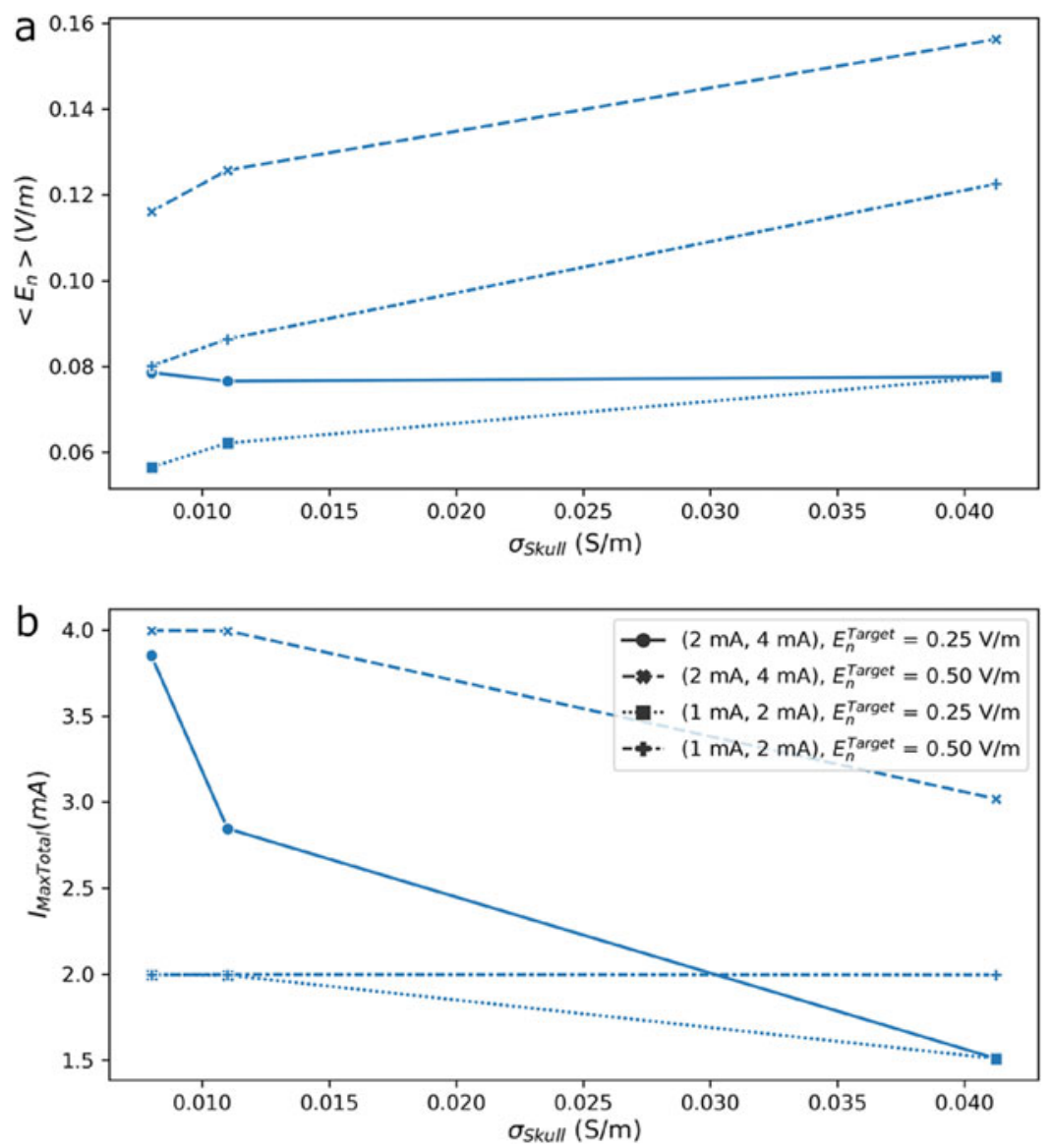

Fig. 4 Average value of $E_{n}$ (in V/m) in the $\operatorname{DLPFC}(\mathbf{a})$ and total injected current $\left(I_{\max \text { total }}\right.$ in $\mathrm{mA}$, b) of the optimized montage as a function of skull conductivity (in $\mathrm{S} / \mathrm{m}$ ). The optimizations were obtained for four different constraints

optimization was then evaluated not only on the subject's head model from which it was derived but also in all the remaining head models. For each evaluation, we calculated the average $\left\langle E_{n}\right\rangle$ value on the IDLPFC. To test the homoscedasticity of the different distributions, we used Levene's test. To compare the means of the different groups, we used Welch's t-test.

As shown in Fig. 6, the variance of $\left\langle E_{n}\right\rangle$ across subjects was significantly lower when using a personalized montage as opposed to a non-personalized montage (Levene's test, p-value $<0.05$ ), except in the most stringent optimization (lowest current constraints with the highest $E_{n}{ }^{\text {Target }}$, Levene's test p-value $=0.73$ ). As is also shown in the figure, no statistically significant difference was found between personalized and non-personalized montages when it comes to the group average of $<E_{n}>$ value across subjects (Welch's t-test p-value $>0.651$ ), when the target $E_{n}$ is 


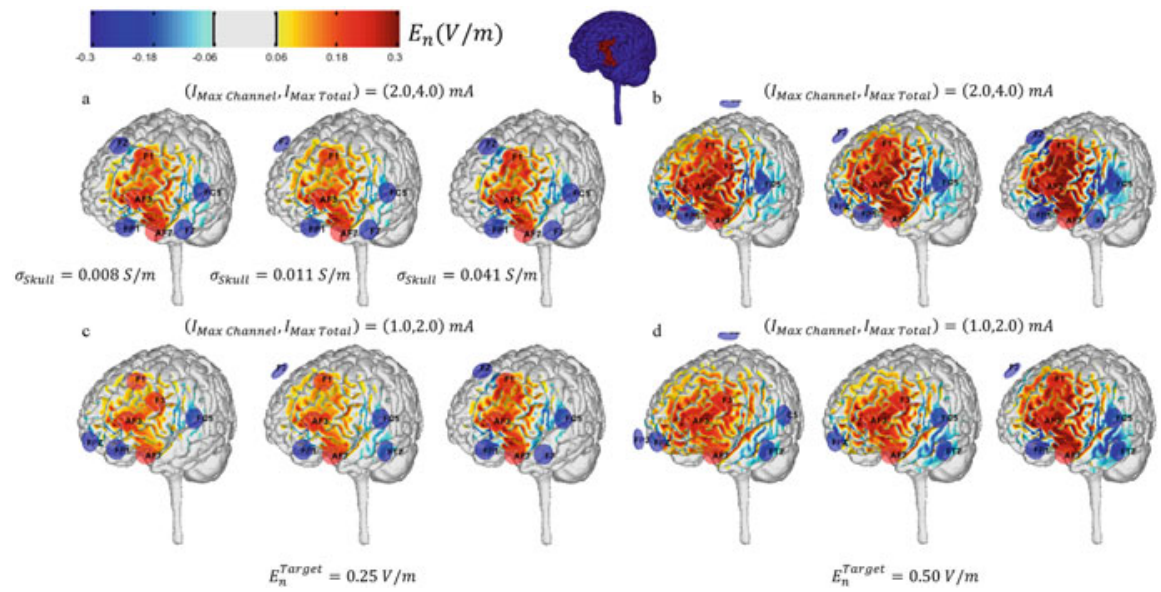

Fig. 5 Distribution of the normal component of the E-field in the cortical surface induced by different montages optimized to increase the excitability of the IDLPFC (shown as an inset in a) as a function of skull conductivity. The current constraints $\left(I_{\text {max channel }}, I_{\text {max total }}\right)$ in each optimization as well as the target $E_{n}$-field $\left(E_{n}{ }^{\text {Target }}\right)$ are shown next to each group of images (a, b, c, and d). The order of the conductivities of the skull within each group of images is the same (see a). The montages were limited to eight channels. The color scale is common to all plots

maintained constant. Increasing the target $E_{n}$ leads to a statistically significant higher $<E_{n}>$, regardless of the current constraints (Welch's t-test p-value $<7.6 \times 10^{-4}$ ).

\section{Discussion}

\subsection{Interplay of Target Size, Cortical Geometry, and Optimization Constraints}

When analyzing the influence of target size and optimization constraints, we found an expected decrease of $\left\langle E_{n}\right\rangle$ with the target area. As mentioned before, the non-monotonous nature of the decrease could be attributed to the interplay of different parameters: cortical geometry, positions of the electrodes available for the optimization, and optimization parameters (current constraints and target $E_{n^{-}}$ field). For small targets that do not encompass multiple sulci and/or gyrus, it was possible to achieve even the highest average $\left\langle E_{n}\right\rangle$ value $(0.5 \mathrm{~V} / \mathrm{m})$ provided enough (total injected) current was set as a limit. Limiting the currents to the safety values used in most studies [28], $\left(I_{\text {max channel }}, I_{\text {max total }}\right)=(2.0,4.0 \mathrm{~mA})$, even $\left\langle E_{n}\right\rangle$ values of $0.25 \mathrm{~V} / \mathrm{m}$ cannot be achieved. This depends of course on target position and electrode array. For instance, at the bottom of the sulci under some of the electrode positions available, local maxima have been shown to be created due to the funneling effect of the CSF layer [15]. In these regions, higher $\left\langle E_{n}\right\rangle$ values might be 

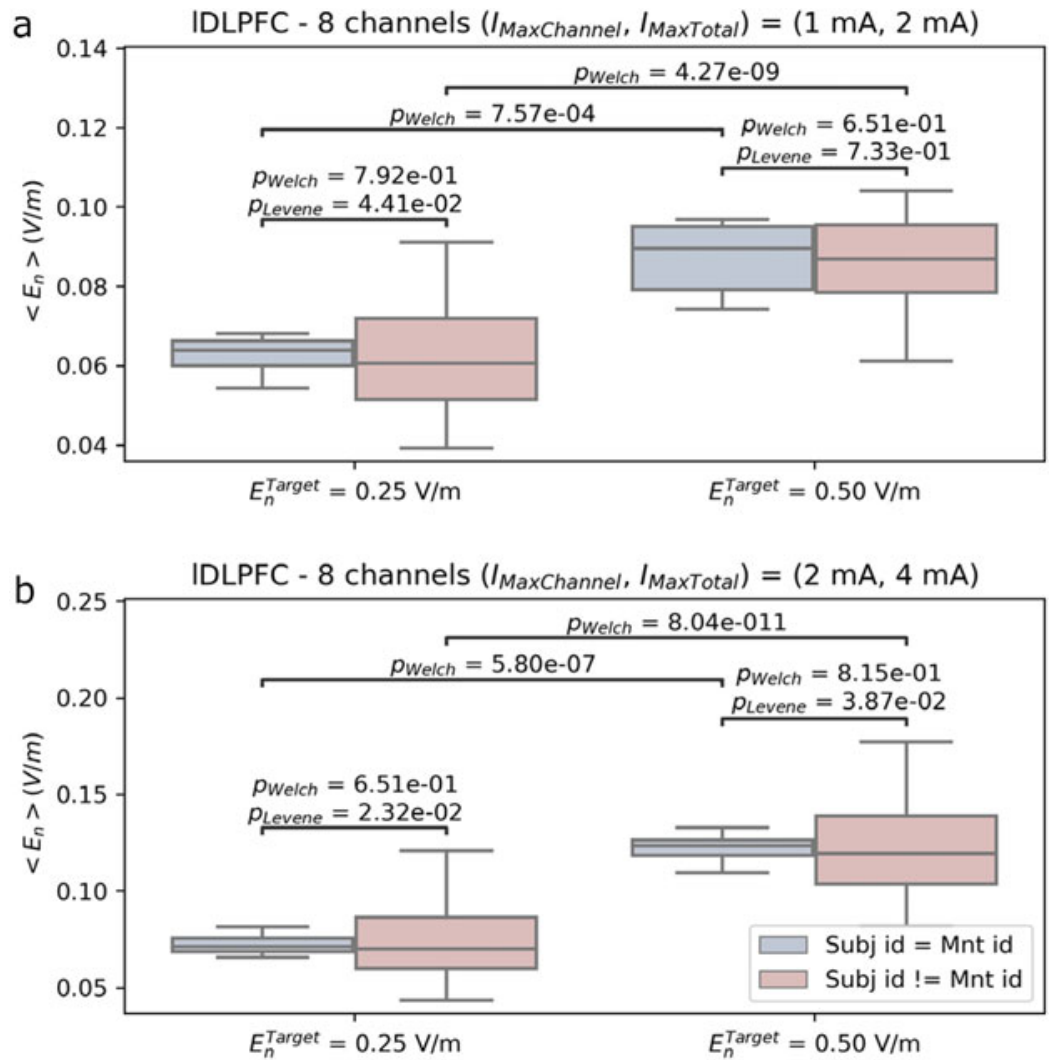

Fig. 6 Effect of current constraints and target $E_{n}$ value on $\left\langle E_{n}\right\rangle$ calculated on the 1DLPFC for personalized (blue) and non-personalized (pink) optimized montages. The top plots (a) show the results for the $\left(I_{\text {max channel }}, I_{\text {max total }}\right)=(1.0,2.0) \mathrm{mA}$ current constraints, whereas the bottom plots (b) show the results for the $\left(I_{\text {max channel }}, I_{\text {max total }}\right)=(2.0,4.0) \mathrm{mA}$ constraints. Welch's t-tests were performed for comparisons between the means of the different groups. Levene's tests were performed to test for homoscedasticity between groups with the same constraints

achievable there with the same current constraints. For these small targets, we also found that the constraint on $I_{\text {max channel }}$ can limit the achievable $<E_{n}>$, with higher values being possible when $I_{\text {max channel }}$ is set to the same value as $I_{\text {max total }}$. For larger targets, the maximum achievable $\left\langle E_{n}\right\rangle$ decreases, firstly due to the folded nature of the cortical surface and the electrode distribution (as shown by the rapid decay of $\left\langle E_{n}\right\rangle$ even with unconstrained currents) and then to the current limitations. In particular, $I_{\text {max total }}$ is the main limiting factor to $\left\langle E_{n}\right\rangle$, with the constraint on $I_{\max }$ channel not mattering as much. This is expected, as larger targets require the distribution of the current on more electrodes to cover the whole area and achieve the same $E_{n}$.

Although the effects of having a more dense electrode array available for the optimization were not tested in this study, it is likely that they would be more 
beneficial for smaller targets (see also [19]), allowing for higher $\left\langle E_{n}>\right.$ to be achieved for the same current constraints.

\subsection{Influence of Skull Conductivity}

The influence of the conductivity of the skull and other tissues on the E-field distribution in tCS is a well-established fact [29, 30]. Provided enough current was available to the optimization algorithm, all models reached a similar $\left\langle E_{n}\right\rangle$ value on target. For more stringent constraints (lower currents and/or higher target $E_{n}$-fields), it might not be possible to maintain a similar $\left\langle E_{n}\right\rangle$ across models (this will again depend on target size). For the latter optimizations, we found that the selected montage employs higher currents and a bigger separation between anodes and cathodes to increase $\left\langle E_{n}\right\rangle$.

In a more realistic scenario, however, the discrepancy between the subject's skull conductivity and the one employed in the model is the main concern. As our results indicate, this can lead to very big discrepancies between the planned and effective $\left\langle E_{n}\right\rangle$. These results stress the need for assessing subject-specific tissue conductivity values and use them together with subject-specific computational head models.

\subsection{Montage Optimization and Intersubject Variability}

Consistent with previously published studies [16], we found a large variability when calculating $\left\langle E_{n}\right\rangle$ induced in six head models by non-personalized montages (on average the standard deviation was $21 \%$ of the mean value across all cases). Employing personalized montages significantly reduced the variation (standard deviation of $8 \%$ of the mean) in all cases except in the more stringent optimization (top-right boxes in Fig. 6). In that case $\left(\left(I_{\max \text { channel }}, I_{\text {max total }}\right)=(1.0,2.0) \mathrm{mA}\right.$ and $E_{n}^{\text {Target }}$ of $\left.0.50 \mathrm{~V} / \mathrm{m}\right)$, personalization of montages did not reduce variability or result in an increase in average $\left\langle E_{n}\right\rangle$ at the target. We interpret this as basically showing that the posed optimization problem is very hard to achieve and hence of variable results even with personalization. Increasing the target $E_{n}{ }^{\text {Target }}$ to $0.50 \mathrm{~V} / \mathrm{m}$ does result in a significant increase in $\left\langle E_{n}\right\rangle$ for both current constraints. Ultimately, this may prove to be more important than decreasing the variability of the results. Heterogeneity in $\left\langle E_{n}\right\rangle$ across subjects can always be used as a regressor when analyzing the results of the study (see [31]). 


\subsection{Study Limitations}

As all studies involving computational head models, there are a number of limitations in this study related to the simplifications employed by the models. The biggest simplification is the adoption of a homogeneous compartment for the skull tissue, ignoring the spongy bone region [32]. Although this would certainly influence the $\left\langle E_{n}\right\rangle$ values reported here, as well as affect the effects of the different current constraints, it is unlikely that the overall qualitative conclusions of the different studies would be influenced.

Another limitation is related to the fact that we focused exclusively on the normal component of the E-field for optimization. The optimization method employed in this study can easily be used with other E-field components, but the optimized montages would employ electrode positions very different from the ones reported here. Again, this is unlikely to affect the overall conclusions of this study, and its general recommendations can be extended when other components of the E-field are of interest.

Regarding the targets, we only considered connected single target regions, despite the fact that interest has arisen lately regarding applications involving multiple distributed targets (as the ones arising from cortical networks, [5, 24]). Again, the stimulation protocol can be readily adapted to these types of targets (with the weights reflecting the statistical significance of the correlation). The conclusions about the influence of current constraints in these types of optimizations are likely to be similar to the ones reported here for the larger areas, but further studies are required.

Finally, we should mention the small number of subjects employed in this study, which limits the generalizability of its results, especially in study c. Future studies are underway which will investigate these findings in a larger population.

\subsection{Consequences for Protocol Design}

The results presented here clearly demonstrate the advantages of employing optimized montages for determining dose parameters in a tCS protocol. They have the potential of reducing variability in the E-field distribution across subjects in a study by taking into effect idiosyncratic subject properties, such as individual head anatomy, electrical properties, and even target location. Of course, these improvements require availability of appropriate data, such as MRI scans with parameters optimized for tissue segmentation [33], protocols for noninvasive determination of tissue electrical conductivities in a fast and reliable way [34, 35], as well as a combination of functional and structural data to determine the target for optimization. Regarding target size and location, this should guide the determination of the electrical current constraints of the study, as is clearly illustrated by the previous results.

Another important limitation of the usefulness of montage optimization is the lack of information about the mechanisms of tCS. However, several studies have 
been published illustrating the interaction of the E-field with neurons [10] and the network amplification effects that can be responsible for the ultimate long-term effects of the intervention [4]. The next step in developing montage optimization protocols would be to combine information about biophysical aspects of current propagation and electrophysiological aspects of E-field - neuron interaction and neuron-neuron communication [5, 36, 37].

Acknowledgments This project has received funding from the European Union's Horizon 2020 research and innovation program under grant agreement No 731827 (project STIPED) and from the European FET Open project Luminous (European Union's Horizon 2020 research and innovation program under grant agreement No 686764). The results and conclusions in this article present the authors' own views and do not reflect those of the EU Commission.

\section{References}

1. Woods, A. J., Antal, A., Bikson, M., Boggio, P. S., Brunoni, A. R., Celnik, P., Cohen, L. G., Fregni, F., Herrmann, C. S., Kappenman, E. S., Knotkova, H., Liebetanz, D., Miniussi, C., Miranda, P. C., Paulus, W., Priori, A., Reato, D., Stagg, C., Wenderoth, N., \& Nitsche, M. A. (2016). A technical guide to tDCS, and related non-invasive brain stimulation tools. Clinical Neurophysiology, 127, 1031-1048.

2. Ruffini, G., Wendling, F., Merlet, I., Molaee-Ardekani, B., Mekkonen, A., Salvador, R., SoriaFrisch, A., Grau, C., Dunne, S., \& Miranda, P. C. (2013). Transcranial current brain stimulation (tCS): Models and technologies. IEEE Transactions on Neural Systems and Rehabilitation Engineering, 21(3), 333-345.

3. Lefaucheur, J.-P., \& Wendling, F. (2019). Mechanisms of action of tDCS: A brief and practical overview. Neurophysiologie Clinique, 49(4), 269-275.

4. Reato, D., Rahman, A., Bikson, M., \& Parra, L. C. (2013, October). Effects of weak transcranial alternating current stimulation on brain activity-a review of known mechanisms from animal studies. Frontiers in Human Neuroscience, 7, 1-8.

5. Ruffini, G., Wendling, F., Sanchez-Todo, R., \& Santarnecchi, E. (2018). Targeting brain networks with multichannel transcranial current stimulation (tCS). Current Opinion in Biomedical Engineering, 8, 70-77.

6. Lefaucheur, J. P., Antal, A., Ayache, S. S., Benninger, D. H., Brunelin, J., Cogiamanian, F., Cotelli, M., De Ridder, D., Ferrucci, R., Langguth, B., Marangolo, P., Mylius, V., Nitsche, M. A., Padberg, F., Palm, U., Poulet, E., Priori, A., Rossi, S., Schecklmann, M., Vanneste, S., Ziemann, U., Garcia-Larrea, L., \& Paulus, W. (2017). Evidence-based guidelines on the therapeutic use of transcranial direct current stimulation (tDCS). Clinical Neurophysiology, $128(1), 56-92$.

7. Polanía, R., Nitsche, M. A., \& Ruff, C. C. (2018). Studying and modifying brain function with non-invasive brain stimulation. Nature Neuroscience, 21(2), 174-187.

8. Radman, T., Ramos, R. L., Brumberg, J. C., \& Bikson, M. (2009). Role of cortical cell type and morphology in subthreshold and suprathreshold uniform electric field stimulation in vitro. Brain Stimulation, 2(4), 215-228.

9. Ruffini, G., Fox, M. D., Ripolles, O., Miranda, P. C., \& Pascual-Leone, A. (2014, April). Optimization of multifocal transcranial current stimulation for weighted cortical pattern targeting from realistic modeling of electric fields. NeuroImage, 89, 216-225.

10. Rahman, A., Reato, D., Arlotti, M., Gasca, F., Datta, A., Parra, L. C., \& Bikson, M. (2013). Cellular effects of acute direct current stimulation: Somatic and synaptic terminal effects. Journal of Physiology (London), 591(10), 2563-2578. 
11. Miranda, P. C., Lomarev, M., \& Hallett, M. (2006). Modeling the current distribution during transcranial direct current stimulation. Clinical Neurophysiology, 117(7), 1623-1629.

12. Huang, Y., Lafon, B., Bikson, M., Parra, L. C., Liu, A. A., Friedman, D., Wang, X., Doyle, W. K., Devinsky, O., \& Dayan, M. (2017). Measurements and models of electric fields in the in vivo human brain during transcranial electric stimulation. eLife, 6, 1-26.

13. Opitz, A., Falchier, A., Yan, C. G., Yeagle, E. M., Linn, G. S., Megevand, P., Thielscher, A., Deborah, A. R., Milham, M. P., Mehta, A. D., \& Schroeder, C. E. (2016). Spatiotemporal structure of intracranial electric fields induced by transcranial electric stimulation in humans and nonhuman primates. Scientific Reports, 6, 31236.

14. Datta, A., Bansal, V., Diaz, J., Patel, J., Reato, D., \& Bikson, M. (2009). Gyri-precise head model of transcranial direct current stimulation: Improved spatial focality using a ring electrode versus conventional rectangular pad. Brain Stimulation, 2(4), 201-207.

15. Miranda, P. C., Mekonnen, A., Salvador, R., \& Ruffini, G. (2013, April). The electric field in the cortex during transcranial current stimulation. NeuroImage, 70, 48-58.

16. Laakso, I., Tanaka, S., Koyama, S., De Santis, V., \& Hirata, A. (2015). Inter-subject variability in electric fields of motor cortical tDCS. Brain Stimulation, 8(5), 906-913.

17. Dmochowski, J. P., Datta, A., Bikson, M., Su, Y. Z., \& Parra, L. C. (2011). Optimized multielectrode stimulation increases focality and intensity at target. Journal of Neural Engineering, $8,4$.

18. Guler, S., Dannhauer, M., Erem, B., Macleod, R., Tucker, D., Turovets, S., Luu, P., Erdogmus, D., \& Brooks, D. H. (2016). Optimization of focality and direction in dense electrode array transcranial direct current stimulation (tDCS). Journal of Neural Engineering, 13(3), 1-31.

19. Saturnino, G. B., Siebner, H. R., Thielscher, A., \& Madsen, K. H. (2019). Accessibility of cortical regions to focal TES: Dependence on spatial position, safety, and practical constraints. NeuroImage, 203, 116183.

20. Wagner, S., Burger, M., \& Wolters, C. H. (2016). An optimization approach for well-targeted transcranial direct current stimulation. SIAM Journal of Applied Mathematics, 76(6), 2154-2174.

21. Fischl, B., Salat, D. H., Busa, E., Albert, M., Dieterich, M., Haselgrove, C., Van Der Kouwe, A., Killiany, R., Kennedy, D., Klaveness, S., Montillo, A., Makris, N., Rosen, B., \& Dale, A. M. (2002). Whole brain segmentation: Automated labeling of neuroanatomical structures in the human brain. Neuron, 33, 341-355.

22. Opitz, A., Windhoff, M., Heidemann, R. M., Turner, R., \& Thielscher, A. (2011). How the brain tissue shapes the electric field induced by transcranial magnetic stimulation. NeuroImage, 58(3), 849-859.

23. Neri, F., Mencarelli, L., Menardi, A., Giovannelli, F., Rossi, S., Sprugnoli, G., Rossi, A., Pascual-leone, A., Salvador, R., Ruffini, G., \& Santarnecchi, E. (2019). A novel tDCS sham approach based on model-driven controlled shunting. Brain Stimulation, 13(2), 507-516.

24. Fischer, D. B., Fried, P. J., Ruffini, G., Ripolles, O., Salvador, R., Banus, J., Ketchabaw, W. T., Santarnecchi, E., Pascual-Leone, A., \& Fox, M. D. (2017). Multifocal tDCS targeting the resting state motor network increases cortical excitability beyond traditional tDCS targeting unilateral motor cortex. NeuroImage, 157, 34-44.

25. Wagner, T., Eden, U., Rushmore, J., Russo, C. J., Dipietro, L., Fregni, F., Simon, S., Rotman, S., Pitskel, N. B., Ramos-Estebanez, C., Pascual-Leone, A., Grodzinsky, A. J., Zahn, M., \& Valero-Cabre, A. (2014). Impact of brain tissue filtering on neurostimulation fields: A modeling study. NeuroImage, 85(Pt 3), 1048-1057.

26. Wendel, K., Väisänen, J., Seemann, G., Hyttinen, J., \& Malmivuo, J. (2010). The influence of age and skull conductivity on surface and subdermal bipolar EEG leads. Computational Intelligence and Neuroscience, 2010, 1-7.

27. Petrides, M., \& Pandya, D. N. (1999). Dorsolateral prefrontal cortex: Comparative cytoarchitectonic analysis in the human and the macaque brain and corticocortical connection patterns. European Journal of Neuroscience, 11, 1-2. 
28. Bikson, M., Grossman, P., Thomas, C., Zannou, A. L., Jiang, J., Adnan, T., Mourdoukoutas, A. P., Kronberg, G., Truong, D., Boggio, P., Brunoni, A. R., Charvet, L., Fregni, F., Fritsch, B., Gillick, B., Hamilton, R. H., Hampstead, B. M., Jankord, R., Kirton, A., Knotkova, H., Liebetanz, D., Liu, A., Loo, C., Nitsche, M. A., Reis, J., Richardson, J. D., Rotenberg, A., Turkeltaub, P. E., \& Woods, A. J. (2016). Safety of transcranial direct current stimulation: Evidence based update 2016. Brain Stimulation, 9, 641-661.

29. Saturnino, G. B., Thielscher, A., Madsen, K. H., Knösche, T. R., \& Weise, K. (2019). A principled approach to conductivity uncertainty analysis in electric field calculations. NeuroImage, 188, 821-834.

30. Salvador, R., Ramirez, F., V'yacheslavovna, M., \& Miranda, P. C. (2012) Effects of tissue dielectric properties on the electric field induced in tDCS: A sensitivity analysis. In: 34th Annual International Conference of the IEEE Engineering in Medicine and Biology Society (EMBC), pp. 787-790.

31. Laakso, I., Mikkonen, M., Koyama, S., Hirata, A., \& Tanaka, S. (2019). Can electric fields explain inter-individual variability in transcranial direct current stimulation of the motor cortex? Scientific Reports, 9, 626.

32. Opitz, A., Paulus, W., Will, S., Antunes, A., \& Thielscher, A. (2015). Determinants of the electric field during transcranial direct current stimulation. NeuroImage, 109, 140-150.

33. Windhoff, M., Opitz, A., \& Thielscher, A. (2013). Electric field calculations in brain stimulation based on finite elements: An optimized processing pipeline for the generation and usage of accurate individual head models. Human Brain Mapping, 34, 923-935.

34. Aydin, U., Rampp, S., Wollbrink, A., Kugel, H., Cho, J., Knosche, T. R., Grova, C., Wellmer, J., \& Wolters, C. H. (2017). Zoomed MRI guided by combined EEG/MEG source analysis: A multimodal approach for optimizing presurgical epilepsy work-up and its application in a multifocal epilepsy patient case study. Brain Topography, 30, 417-433.

35. Fernandez-Corazza, M., Turovets, S., Luu, P., Price, N., Muravchik, C. H., \& Tucker, D. (2018). Skull modeling effects in conductivity estimates using parametric electrical impedance tomography. IEEE Transactions on Biomedical Engineering, 65, 1785-1797.

36. Sanchez-Todo, R., Salvador, R. Santarnecchi, E., Wendling, F., Deco, G., \& Ruffini, G. (2018). Personalization of hybrid brain models from neuroimaging and electrophysiology data. bioRxiv 461350 [Preprint].

37. Ruffini, G., Salvador, R., Tadayon, E., Sanchez-Todo, R., Pascual-Leone, A., \& Santarnecchi, E. (2019). Realistic modeling of ephaptic fields in the human brain, bioRxiv, 688101 [Preprint].

Open Access This chapter is licensed under the terms of the Creative Commons Attribution 4.0 International License (http://creativecommons.org/licenses/by/4.0/), which permits use, sharing, adaptation, distribution and reproduction in any medium or format, as long as you give appropriate credit to the original author(s) and the source, provide a link to the Creative Commons license and indicate if changes were made.

The images or other third party material in this chapter are included in the chapter's Creative Commons license, unless indicated otherwise in a credit line to the material. If material is not included in the chapter's Creative Commons license and your intended use is not permitted by statutory regulation or exceeds the permitted use, you will need to obtain permission directly from the copyright holder.

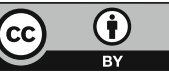

